\title{
Is hyperkyphosis associated with increased mortality in the elderly population?
}

\author{
“These latest study results illustrate a combined ill effect \\ of vertebral fractures and hyperkyphosis on health, and signal that \\ clinicians should pay more attention not only to osteoporosis, but also \\ to age-related postural changes."
}

Hyperkyphosis, or an abnormally increased spinal curve in the mid-back, has been a largely ignored clinical finding in the aging population. In fact, other than its colloquial name of 'dowager's hump', which confers the image of an elderly, hunched-over woman, there is discrepancy in the medical terminology used to describe this condition. Some use the term 'kyphotic' to characterize a patient with accentuated thoracic spine curvature; however, just as there is normal lordosis that refers to the natural convex curvature of the lower lumbar spine, there is normal kyphosis. Therefore, in order to differentiate normal from abnormal, hyperkyphosis and kyphosis, rather than hyperkyphotic and kyphotic, are the preferred terms to use when describing patients with accentuated thoracic spinal curvature.

“Women who had worse degrees of kyphosis had an increased risk of earlier mortality..."

Up until now, other than being cosmetically undesirable, age-related hyperkyphosis has not garnered much attention. Most physicians and patients have largely equated this problem with underlying vertebral fractures, and few researchers have focused on hyperkyphosis as a separate and important clinical entity. However, a recently published article in the Annals of Internal Medicine in May 2009 confirms earlier reports that hyperkyphosis is associated with earlier death [1-3]. Unlike previous studies, this recent investigation was able to disentangle the effects of vertebral fractures from hyperkyphosis and demonstrate that the forward bent posture itself deserves further clinical consideration [4]. Prior to this publication, underlying vertebral fractures that are a known risk factor for mortality had been assumed to be the explanation for the findings of increased death observed in the previous studies, especially since up to two-thirds of the population with vertebral fractures remain clinically undiagnosed [5]. In this latest study, participants included 610 predominantly White women aged 65 years and older who had their thoracic curvature measured, had lateral spine radiographs that were systematically evaluated for underlying vertebral fractures, and who were followed for an average of 13.5 years. Women who had worse degrees of kyphosis had an increased risk of earlier mortality, an effect that was not accounted for by controlling for age, bone mineral density, vertebral fractures or other factors associated with earlier mortality, such as smoking, history of stroke, weight loss and an inability to stand up and down from a chair five times without the use of the arms. Furthermore, compared with women who had either only vertebral fractures or only hyperkyphosis, the women who suffered from both vertebral fractures and hyperkyphosis were at the greatest risk of earlier mortality.

These latest study results illustrate a combined ill effect of vertebral fractures and hyperkyphosis on health, and signal that clinicians should pay more attention not only to osteoporosis, but also to age-related postural changes. Furthermore, in addition to increased mortality, hyperkyphosis is associated with impaired physical and pulmonary function, injurious falls and fractures [6]. With just one exception [7], these studies were all cross-sectional in design, making it difficult to ascertain whether it was the hyperkyphosis that preceded the poor outcome or vice versa. Evidently, this field of investigation is wide open to investigate whether hyperkyphosis is likely to precede worse health outcomes. If it does, then intervening to prevent worsening kyphosis may make sense in terms of trying to prevent future impaired pulmonary and physical function, for example. For now, what these cross-sectional studies have accomplished is to demonstrate that, regardless of the cause, having hyperkyphosis is not associated with healthy aging.
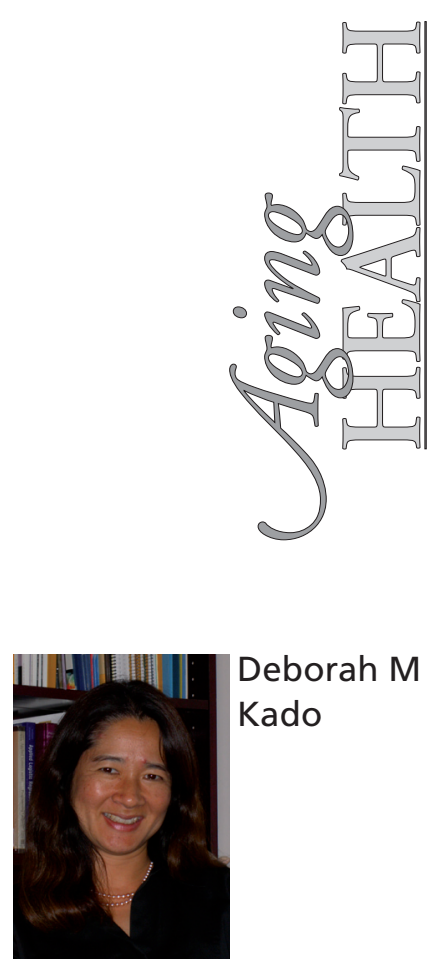

David Geffen School of Medicine at the University of California, Los Angeles, CA 90095, USA Tel.: +1 3108254527 Fax: +1310 2065674 dkado@mednet.ucla.edu

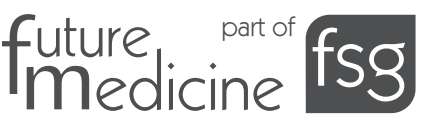


Although the commonly used term to describe hyperkyphosis, dowager's hump, by definition implies that it is a female condition, the name is a misnomer. Older men also suffer from age-related postural changes and, depending on the exact location (whether in the cervical versus thoracic area), older men may be predominantly affected [3]. This observation and the fact that osteoporosis is less common in men than in women, suggests that the causes of age-related postural changes deserve further investigation. In addition, although most studies to date have included only older women with osteoporosis, the few that have included men suggest that men may be at an even greater risk for earlier mortality than older women $[1,3]$. More studies in men would help elucidate the pathogenesis of this age-related condition while also potentially informing targeted strategies for prevention.

"...regardless of the cause, having hyperkyphosis is not associated with healthy aging."

So, is hyperkyphosis associated with mortality in the elderly population? Based upon the evidence to date and not being able to account for possible negative study publication bias, the answer is yes. Perhaps an alternative question might be 'does hyperkyphosis cause earlier mortality?' This answer to this is no. Rather, the association between hyperkyphosis and mortality might be thought of as similar to the associations between hip fractures or delirium and mortality in older persons. Each of these conditions commonly associated with aging is linked with a decline in functional abilities, loss of independence and eventual death. However, with hyperkyphosis, there may be more hope to affect future health outcomes. Hyperkyphosis is an easily detectable clinical finding that generally progresses slowly with age. Unlike hip fractures and delirium, which are hard to diagnose before the event, mild hyperkyphosis is readily apparent to the trained eye. Now that there is increasing awareness of hyperkyphosis, the next steps will be to determine whether preventative strategies can help to prevent future functional decline and earlier mortality in affected persons.

\section{Financial \& competing interests disclosure}

Deborah $M$ Kado receives grant funding from the National Institutes of Health, National Institute on Aging, and serves as a consultant for Medtronic, Octapharma and Amgen. The author has no other relevant affliations or financial involvement with any organization or entity with a financial interest in or financial conflict with the subject matter or materials discussed in the manuscript apart from those disclosed.

No writing assistance was utilized in the production of this manuscript.

\section{Bibliography}

Papers of special note have been highlighted as:

- of interest

- of considerable interest

1. Milne JS, Williamson J: A longitudinal study of kyphosis in older people. Age Ageing 12(3), 225-233 (1983).

2. Kado DM, Browner WS, Palermo L, Nevitt MC, Genant HK, Cummings SR: Vertebral fractures and mortality in older women: a prospective study. Study of Osteoporotic Fractures Research Group. Arch. Intern. Med. 159(11), 1215-1220 (1999).

3. Kado DM, Huang MH, Karlamangla AS, Barrett-Connor E, Greendale GA: Hyperkyphotic posture predicts mortality in older community-dwelling men and women: a prospective study. J. Am. Geriatr. Soc. 52(10), 1662-1667 (2004).
4. Kado DM, Lui LY, Ensrud KE, Fink HA, Karlamangla AS, Cummings SR: Hyperkyphosis predicts mortality independent of vertebral osteoporosis in older women. Ann. Intern. Med. 150 (10), 681-687 (2009).

- Recent investigation that is discussed in this article.

5. Cooper C, Atkinson EJ, O’Fallon WM, Melton LJ 3rd: Incidence of clinically diagnosed vertebral fractures: a populationbased study in Rochester, Minnesota, 1985-1989. J. Bone Miner. Res. 7(2), 221-227 (1992).

6. Kado DM, Prenovost K, Crandall C: Narrative review: hyperkyphosis in older persons. Ann. Intern. Med. 147(5), 330-338 (2007).
- First comprehensive review on hyperkyphosis in older persons that discusses the causes and consequences of hyperkyphosis in older persons.

7. Huang $\mathrm{MH}$, Barrett-Connor E, Greendale GA, Kado DM: Hyperkyphotic posture and risk of future osteoporotic fractures: the Rancho Bernardo study. J. Bone Miner. Res. 21(3), 419-423 (2006).

\section{Affiliation}

- Deborah M Kado, MD, MS David Geffen School of Medicine, University of California, Los Angeles, Los Angeles, CA 90095, USA Tel.: +1 3108254527 Fax: +1 3102065674 dkado@mednet.ucla.edu 